Revista PSICOLOGIA, 2014, Vol. 28 (2), 31-44

\title{
Tradução, adaptação e evidências de validade da versão brasileira do Overexcitability Questionnaire Two
}

\author{
Juliana Célia de Oliveira ${ }^{1}$, Altemir José Gonçalves Barbosa ${ }^{1}$ \\ ${ }^{1}$ Universidade Federal de Juiz de Fora
}

\begin{abstract}
Resumo: Três estudos foram realizados para traduzir, adaptar para a língua portuguesa e analisar as propriedades psicométricas do Overexcitability Questionnaire Two (OEQ-II) - uma medida de cinco padrões de sobre-excitabilidade (Intelectual, Psicomotor, Sensorial, Emocional e Imaginativo). No primeiro estudo, foram efetuadas tradução, retrotradução, análise por juízes e aplicação piloto a fim de obter evidências de validade de conteúdo do OEQ-II. O Estudo 2 contou com a participação de 282 universitários e analisou a confiabilidade e as evidências de validade baseadas na estrutura interna dessa medida. No terceiro estudo, que teve como amostra 368 universitários, almejou-se obter evidências de validade para o OEQ-II baseadas nas relações com medidas que avaliam constructos análogos. Os resultados revelaram que a versão em português desse questionário possui boa confiabilidade e boas evidências de validade. As limitações do estudo e sugestões para pesquisas futuras são apresentadas no artigo.
\end{abstract}

Palavras-chave: Sobredotação; Sobre-Excitabilidade; Avaliação psicológica; Psicometria.

Translation, adaptation and evidence of validity of the Brazilian version of Overexcitability Questionnaire Two: Three studies was performed in order to translate, adapt to Portuguese language and analyze the psychometric properties of the Overexcitability Questionnaire Two (OEQ-II) - a measure of five overexcitabilities patterns (Intellectual, Psychomotor, Sensual, Emotional, and Imaginational). In the first study, translation, back translation, analysis by judges, and pilot application were performed for obtaining validity evidence of content of the OEQ-II. The Study 2 involved the participation of 282 undergraduate students and it was analyzed reliability and validity evidence based on internal structure of the measure. In the third study, which had as sample 368 undergraduate students, it was aimed to obtain for the OEQ-II validity evidence based on relations with measures that assess similar constructs. The results showed that the version of OEQ-II in Portuguese language has good reliability and good validity evidence. Limitations of this study and suggestions for future researches are analyzed in this article.

Keywords: Giftedness; Overexcitability; Psychological assessment; Psychometry.

A área de Avaliação Psicológica tem crescido, nos últimos anos, no Brasil e em outros países, como Portugal, incluindo o desenvolvimento de testes e outras medidas (Diniz, Almeida, \& Pais, 2007; Noronha, Primi, \& Alquiere, 2005; Noronha \& Reppold, 2010; Simões, 2005). No entanto, ainda existem poucos instrumentos com evidências de validade que podem ser usados no processo de identificação de sobredotação. Mesmo no caso dos testes de inteligência - os mais comuns nessa área (Kaufman \& Sternberg, 2008) -, são poucos os instrumentos disponíveis, por exemplo, no Sistema de Avaliação de Testes Psicológicos (SATEPSI) brasileiro [Conselho Federal de Psicologia (CFP), 2013], diferentemente do que ocorre, por exemplo, nos Estados Unidos (Pierson, Kilmer, Rothlisberg, \& McIntosh, 2012). Quando se tratam de medidas que avaliam outros domínios (p.ex., psicomotor) ou que foram desenvolvidas especificamente para processos de identificação de sobredotação, a quantidade é ainda menor (Barbosa, Schelini, \& Almeida, 2012).

Dentre as múltiplas concepções de sobredotação, menciona-se, por exemplo, a de Renzulli (1978, 1998, 2005), que propõe uma representação desse constructo baseada em um diagrama de Venn composto por alta criatividade, potencial elevado em diversas áreas de conhecimento, além de grande motivação para a aprendizagem e realização de atividades de interesse pessoal. Nesta e em outras perspectivas (p.ex., Carroll, 1993; Heller, Perleth, \& Lim, 2005), a psicometria tem desempenhado um

Dados de contacto para correspondência: Juliana Oliveira; Rua Padre Frederico, 337. Santa Catarina. Juiz de Fora. Minas Gerais. Brasil. E-mail: julianaoliveirapsi@gmail.com. 
papel importante na identificação de estudantes com sobredotação, principalmente no que se refere à elaboração e ao uso de instrumentos para a avaliação de inteligência (Kaufman \& Sternberg, 2008). Apesar do amplo uso na pesquisa e na prática em Psicologia Escolar/Educacional, são muitos os estudiosos que assinalam as limitações dos testes de inteligência e de desempenho académico para a identificação de estudantes com sobredotação (Gagné, 1985; Guenther, 2006a; Renzulli, 1978; Soares, Arco-Verde, \& Baibich, 2004; Virgolim, 1997). De modo geral, esses e outros autores alertam que vários domínios de sobredotação são desconsiderados, como, por exemplo, aqueles relacionados com as funções motoras, artísticas e criativas. Além dessas limitações, Carman (2011) acrescenta que, ao privilegiar aspectos intelectuais, essas medidas dificilmente abarcam todos os estudantes igualmente.

Os processos de avaliação de sobredotação devem ser multidimensionais e contínuos ou seja, que não sejam pontuais e ocorram em diferentes momentos (Feldhusen, 1998; Gagné, 2004; Guenther, 2006b; Renzulli, 1978), baseados em múltiplos informantes e múltiplas medidas (Oliveira, 2007). Assim, os testes psicológicos representam um dos componentes do processo de identificação, não sendo, portanto, a única medida. Não obstante, eles têm, historicamente, desempenhado um papel importante nessa área (Kaufman \& Sternberg, 2008) e, portanto, devem ser escolhidos criteriosamente, considerando, por exemplo, suas evidências de validade.

A sobre-excitabilidade psíquica (SE) tem sido considerada um indicador confiável de sobredotação (Ackerman, 1997; Carman, 2011; Siu, 2010; Tieso, 2007a) e vários instrumentos têm sido desenvolvidos para avaliá-la, tais como o Overexcitability Questionnaire (Lisy \& Piechowski, 1983; Piechowski, 1996), a Me Scale (Chang \& Kuo, 2009), o ElemenOE (Bouchard, 2004) e o Overexcitability Questionnaire Two (OEQ-II) (Falk, Lind, Miller, Piechowski, \& Silverman, 1999). A SE é um atributo da personalidade e diz respeito a uma forma ampliada e intensificada do indivíduo reagir, podendo ocorrer em um ou mais dos seguintes padrões: Sensorial, Psicomotor, Intelectual, Imaginativo e Emocional (Dabrowski, 1972). Esse constructo tem-se destacado no âmbito internacional, pois extrapola o conceito de sobredotação para além do domínio intelectual.

Os cinco padrões de SE foram detalhados por Dabrowski $(1972,1996)$ e outros pesquisadores (Falk et. al, 1999; Piechowski, 1975). Para eles, a SE Psicomotora tende a ser expressa por indivíduos intensamente ativos, agitados, impulsivos e com dificuldade em se manter parados. A SE Sensorial diz respeito à elevada diferenciação e vivacidade de experiências sensoriais, como as percebidas em componentes estéticos de objetos através de cores, formas, texturas, aromas, sons etc. Indivíduos com SE Imaginativa apresentam pensamentos criativos, inusitados e têm maior facilidade para fantasiar e/ou sonhar, realizar criações poéticas, inventar histórias etc. A SE Intelectual é manifestada pela independência de pensamento, interesse e curiosidade por conhecimento e pela persistência em buscar soluções para diversos problemas. 0 padrão de SE Emocional evidencia-se em indivíduos que apresentam forte apego a pessoas, seres vivos e/ou lugares, com uma gama diferenciada de sentimentos, afetos, paixões, senso de responsabilidade e empatia.

De acordo com Dabrowski (1972), indivíduos que apresentam padrões de respostas muito intensos experimentam o mundo de forma diferenciada, com mais energia, qualitativa e quantitativamente. Em suas pesquisas e estudos clínicos, o autor e seus colaboradores chegaram à conclusão de que tais características são, também, aspectos encontrados em pessoas criativas e com sobredotação (Dabrowski, Kawczak, \& Piechowski, 1970). Posteriormente, Piechowski (1979) acrescentou que as SEs são componentes básicos da sobredotação, compartilhadas por muitos indivíduos talentosos. Desde então, diversos pesquisadores têm testado a hipótese de que SE é um dos preditores de sobredotação, corroborando-a (Ackerman, 1997; Bouchet \& Falk, 2001; Tieso, 2007a).

Reitera-se que, devido as SEs representarem indicadores confiáveis de sobredotação, construir medidas que avaliem esses padrões pode auxiliar no processo de identificação de estudantes com essas características. O OEQ-II (Falk et al., 1999), por exemplo, destaca-se como a medida de SE mais utilizada para identificar sobredotação (Bouchet \& Falk, 2001; Carman, 2011; Siu, 2010; Tieso, 2007a; Wirthwein \& Rost, 2011) e é a que apresenta mais evidências de validade (Carman, 2011; Gross, Rimm, \& Jamieson, 2007; Tieso, 2007a, 2007b; Wirthwein \& Rost, 2011). Em sua versão original, as análises da aplicação do OEQ-II em estudantes universitários demonstraram que os itens do instrumento apresentaram boa validade de conteúdo e de constructo. Uma estrutura de cinco fatores foi obtida, cada um com alta confiabilidade, variando de .84 a .89. Estudos posteriores têm confirmado a estrutura multidimensional da medida (Sanz, 2006; Warne, 2011a), corroborando que, apesar de poderem ser associadas, as SEs são teoricamente independentes umas das outras (Ackerman, 2009).

Destaca-se, além disso, que o OEQ-II tem sido traduzido, adaptado e validado para diversos idiomas e países, tais como Espanha (Sanz, 2006), Coreia do Sul (Piirto, Montgomery \& May, 2008), Hong Kong (Siu, 2010), Jordânia (Al-Onizat, 2013), Turquia, México (Falk, Yakmaci-Guzel, Chang, Sanz, \& Chavez-Eakle, 2008) etc., o que denota a validade intercultural do constructo SE e do instrumento. Os 
estudos de confiabilidade da medida demonstram uma variação de .57 a .91 no alfa de Conbrach nos diferentes padrões de SE. Salienta-se que a maioria dos estudos obteve "alfas" superiores a .80 (Warne, 2011b). Para obter mais detalhes sobre as análises da confiabilidade do instrumento, recomenda-se que sejam consultadas, por exemplo, as publicações de Falk et al. (2008) e Warne (2011b).

As análises das evidências de validade de constructo e de critério sobressaem nas investigações que utilizaram o OEQ-II (Oliveira \& Barbosa, 2014). Entretanto, são poucas as que procuraram observar a correlação do OEQ-II com outras medidas. Carman (2011), por exemplo, enfatiza como aspecto negativo do instrumento o fato de ele não possuir evidências de validade convergente com testes "importantes", apesar de uma iniciativa ter sido feita por essa autora ao comparar os padrões Sensorial e Psicomotor ao Sensory Profile - uma medida de análise do processamento sensorial no desempenho funcional proposto por Dunn (1997), obtendo correlações positivas entre os dois.

Apesar do destaque do OEQ-II em âmbito internacional, este instrumento ainda não foi traduzido para a língua portuguesa. Nesse sentido, este artigo apresenta três estudos que tiveram como objetivo geral traduzir e adaptar uma medida de SE - OEQ-II -, bem como analisar as propriedades psicométricas dessa medida. Especificamente, no Estudo 1 almejou-se apresentar evidências de validade de conteúdo obtidas a partir do processo de tradução e retrotradução, da análise por juízes e de uma aplicação piloto. o Estudo 2, que contou com estudantes universitários, teve como objetivo obter evidências de validade baseadas na estrutura interna (validade fatorial) e confiabilidade da versão brasileira do instrumento. Por fim, o Estudo 3, que contou com uma segunda amostra de universitários, objetivou obter evidências de validade adicionais da medida por meio da correlação com instrumentos que investigam constructos correlatos.

\section{ESTUDO 1 - EVIDÊNCIAS DE VALIDADE BASEADAS NO CONTEÚDO DO OEQ-II}

\section{MÉTODO \\ Participantes}

Neste Estudo, três grupos de participantes podem ser distinguidos. 0 primeiro grupo refere-se aos juízes doutores $(\mathrm{N}=6)$ - proficientes em língua inglesa e especialistas em sobredotação - que participaram do processo de tradução do OEQ-II para a língua portuguesa, bem como avaliaram a adequação dos itens aos padrões de SE.

Uma segunda amostra composta por estudantes $(n=30)$ do terceiro ano do Ensino Médio de uma escola pública participou de uma aplicação piloto. A idade média em anos desses alunos foi igual a 17.20 $(D P=0,92)$, sendo que $22(73.30 \%)$ eram do sexo feminino. Essa amostra, obtida por conveniência, foi escolhida por se tratar de alunos com um nível de escolaridade igual ao recomendado para o autopreenchimento do OEQ-II, ou seja, superior ao nono ano do Ensino Fundamental. Além disso, supôsse que, se esses discentes conseguissem entender adequadamente os itens e as instruções do questionário, os estudantes universitários que compuseram as amostras dos dois outros estudos apresentados neste artigo não teriam dificuldade para preenchê-lo.

0 terceiro grupo de participantes analisou a adequação dos itens aos padrões de SE. Ele foi composto por 20 mestrandos matriculados em um programa de pós-graduação em Psicologia de uma universidade brasileira e escolhidos por se tratar de um conjunto de profissionais diretamente relacionados à área de avaliação psicológica, mas que podiam atuar como juízes 'cegos' por não terem familiaridade com o tema SE.

\section{Instrumentos}

0 instrumento analisado no presente estudo diz respeito à versão em português do OEQ-II. Trata-se de um instrumento de auto-avaliação, composto por 50 itens respondidos em uma escala Likert de cinco pontos, variando de um - "não se parece nada comigo" - a cinco - "se parece totalmente comigo". As afirmações são precedidas por um enunciado bastante simples, o qual solicita que, ao preencher, a pessoa considere o quanto cada uma delas reflete a forma como se vê. 0 questionário permite obter cinco escores referentes às formas de SE, sendo 10 itens para cada padrão: Psicomotor (itens 2, 7, 10, 15, 18, $21,29,39$, 42 e 50), Sensorial (itens 3, 8, 13, 27, 32, 37, 38, 45, 46 e 48), Imaginativo (itens 1, 4, 14, 20, 22 , 24, 28, 33, 34 e 47), Intelectual (itens 5, 12, 16, 19, 23, 25, 30, 36, 40 e 43) e Emocional (itens 6, 9, 11, 17, $26,31,35,41,44$ e 49). Para computar cada padrão, é preciso somar os pontos obtidos em cada item e dividir por dez. No caso dos itens 38 e 44, é preciso espelhar a pontuação antes de efetuar a adição, pois tratam-se de itens formulados negativamente (Falk et al., 1999).

Para a análise por juízes doutores, utilizou-se um formulário dividido em quatro partes, que se iniciava com uma breve descrição de cada padrão de SE. A primeira parte, continha os itens do OEQ-II em inglês; a segunda apresentava a sua tradução inicial para o português; na terceira, havia um espaço para 
que os participantes expressassem concordância ou discordância quanto à tradução realizada; e a última parte apresentava um campo para que o item fosse associado a um dos cinco padrões de SE. Um formulário análogo ao dos juízes doutores foi usado com os mestrandos. Porém, ele não continha o campo destinado à análise da adequação da tradução.

\section{Procedimento}

Destaca-se que todos os estudos relatados no presente artigo cumpriram as exigências éticas para pesquisa com seres humanos, incluindo a aprovação do projeto por um Comitê de Ética. Ressalta-se que foram obtidos termos de consentimento livre e esclarecido ou, no caso dos menores, termo de dissentimento dos responsáveis legais e assentimento oral dos adolescentes antes da coleta de dados.

Inicialmente, a tradução do OEQ-II para a língua portuguesa, realizada pelos autores do presente estudo, foi submetida aos juízes doutores. Estes foram contatados via correio eletrônico e receberam o formulário de análise para juízes. Após as suas análises, a versão brasileira do OEQ-II foi aplicada aos estudantes de Ensino Médio. Foi solicitado aos discentes que, além de preencherem o instrumento, avaliassem oralmente a compreensão dos itens, das instruções e dos pontos de resposta, bem como que tecessem comentários sobre os aspectos gráficos (legibilidade, aparência etc.) do instrumento. Posteriormente, os mestrandos preencheram individualmente o formulário a eles destinado.

Destaca-se que, no que se refere aos procedimentos de análises de dados, especialmente a precisão entre juízes, foi utilizado o coeficiente Kappa, cuja classificação do grau de concordância teve como base os critérios adotados por Landis e Koch (1977). Os autores sugerem a seguinte categorização para os valores de Kappa compreendidos entre: 1.00 a .81, concordância quase perfeita; .80 a .61, concordância substancial; .60 a .41, concordância moderada; .40 a .21, concordância regular; .20 a .00, concordância fraca; e valores inferiores a .00, concordância pobre. Também foi calculado o percentual de concordância dos juízes para cada item de acordo com o padrão de SE estabelecido pelos autores do OEQ-II. A literatura recomenda índice de concordância maior ou igual a 80\% (Pasquali, 2001).

Por fim, uma retrotradução da última versão do questionário foi feita por um profissional fluente em língua inglesa. Esse foi um procedimento cego, pois o tradutor não teve acesso à versão original do questionário. 0 instrumento retrotraduzido foi enviado para um dos autores do questionário a fim de verificar se havia convergência semântica com a versão original em inglês.

\section{RESULTADOS}

\section{Análises por juízes doutores}

Ao comparar as respostas dos juízes, foi possível observar que todos os participantes consideraram a tradução e/ou adaptação para a língua portuguesa adequada em 18 dos 50 itens do questionário (1, 2, 3, $4,10,12,14,15,18,23,29,30,31,34,38,39,40$ e 48). Para esses itens, nenhum juiz sugeriu modificações na estrutura ou semântica das afirmações.

Quatro dos seis doutores opinaram sobre a tradução de algumas frases. Para alguns itens $(6,7,21$, 25, 28, 43, 44 e 46), foram acatadas as sugestões dos juízes (Quadro 1), uma vez que expressavam melhor em português o sentido original do item ou tornavam as frases mais compreensíveis.

Quadro 1. Itens alterados após as análises realizadas pelos juízes doutores.

\begin{tabular}{ll}
\hline \multicolumn{1}{c}{ Tradução inicial dos itens } & \multicolumn{1}{c}{ Itens alterados após as sugestões dos juízes } \\
\hline $\begin{array}{l}\text { 6. Eu sinto o que as outras pessoas sentem. } \\
\text { 7. Se uma atividade é exaustiva fisicamente, eu considero-a } \\
\text { agradável. }\end{array}$ & $\begin{array}{l}\text { 6. Eu sinto os sentimentos de outras pessoas. } \\
\text { 7. Se uma atividade é exaustiva fisicamente, eu a considero } \\
\text { agradável. }\end{array}$ \\
$\begin{array}{l}\text { 21. Se eu tenho que ficar quieto(a) por um tempo, mais } \\
\text { agitado(a) fico. }\end{array}$ & $\begin{array}{l}\text { 21. Quanto mais tenho que ficar quieto(a) mais agitado(a) fico. } \\
\text { 25. Teorias vêm em minha mente. }\end{array}$ \\
$\begin{array}{l}\text { 28. Eu gosto de 'aumentar a realidade'. } \\
\text { 43. Eu gosto de brincar com ideias e de tentar pensar em como fazem a minha mente funcionar. } \\
\text { colocá-las em prática. }\end{array}$ & $\begin{array}{l}\text { 28. Eu gosto de exagerar a realidade. } \\
\text { 44. Eu gosto de brincar com ideias e tento pensar sobre como } \\
\text { colocá-las em prática. }\end{array}$ \\
46. A diferença de aromas é interessante. & 44. Eu não sou uma pessoa emotiva. \\
\end{tabular}

No entanto, para o restante dos itens optou-se pela permanência da tradução inicial. Tal opção se deve ao fato de as sugestões gerarem mudanças substanciais no significado do item original ou não contribuírem para uma melhor compreensão das frases. Além disso, na maioria desses itens $(n=17$; $70,8 \%$ ) somente um juiz, do total de seis, sugeriu alteração.

No que diz respeito à adequação dos itens avaliada pelos participantes, observou-se que os índices de concordância em relação ao padrão esperado, ou seja, como ocorre no OEQ-II, foram classificados de 
substanciais a quase perfeitos, uma vez que os valores obtidos por todos os juízes variaram entre .75 e .95 (com valores de $p<.001$ em todos os casos). No Quadro 2, é apresentada a percentagem de concordância entre os juízes em relação a cada item. Observou-se que, dos 50 itens, apenas cinco (itens 2, 34, 38, 43 e 44) apresentaram concordância abaixo da recomendada (Pasquali, 2001), ou seja, foram inferiores a $80 \%$.

Quadro 2. Índices de concordância entre juízes doutores (JD) e entre os juízes mestrandos (JM) quanto aos itens nos padrões de SE

\begin{tabular}{|c|c|c|c|c|c|c|c|c|c|c|c|c|c|c|}
\hline \multicolumn{3}{|c|}{ Psicomotor } & \multicolumn{3}{|c|}{ Sensorial } & \multicolumn{3}{|c|}{ Imaginativo } & \multicolumn{3}{|c|}{ Intelectual } & \multicolumn{3}{|c|}{ Emocional } \\
\hline Itens & JD (\%) & $\begin{array}{c}\text { JM } \\
(\%)\end{array}$ & Itens & $\begin{array}{c}\text { JD } \\
\text { (\%) }\end{array}$ & $\begin{array}{c}\text { JM } \\
(\%) \\
\end{array}$ & Itens & $\begin{array}{c}\text { JD } \\
\text { (\%) }\end{array}$ & $\begin{array}{c}\text { JM } \\
\text { (\%) }\end{array}$ & Itens & $\begin{array}{c}\text { JD } \\
(\%) \\
\end{array}$ & $\begin{array}{l}\text { JM } \\
(\%) \\
\end{array}$ & Itens & $\begin{array}{c}\text { JD } \\
(\%) \\
\end{array}$ & $\begin{array}{c}\text { JM } \\
\text { (\%) }\end{array}$ \\
\hline 2 & 20 & 95 & 3 & 100 & 100 & 1 & 83.33 & 85 & 5 & 83.33 & 90 & 6 & 100 & 95 \\
\hline 7 & 100 & 90 & 8 & 83.33 & 84.21 & 4 & 100 & 100 & 12 & 100 & 95 & 9 & 80 & 55 \\
\hline 10 & 100 & 95 & 13 & 100 & 95 & 14 & 100 & 85 & 16 & 100 & 100 & 11 & 100 & 100 \\
\hline 15 & 100 & 100 & 27 & 100 & 85 & 20 & 100 & 100 & 19 & 83.33 & 95 & 17 & 100 & 94.74 \\
\hline 18 & 100 & 100 & 32 & 100 & 100 & 22 & 100 & 95 & 23 & 100 & 95 & 26 & 100 & 90 \\
\hline 21 & 100 & 90 & 37 & 80 & 90 & 24 & 100 & 95 & 25 & 100 & 100 & 31 & 83.33 & 95 \\
\hline 29 & 100 & 95 & 38 & 40 & 70 & 28 & 100 & 85 & 30 & 100 & 100 & 35 & 100 & 95 \\
\hline 39 & 100 & 85 & 45 & 100 & 100 & 33 & 83.33 & 90 & 36 & 100 & 94.74 & 41 & 100 & 70 \\
\hline 42 & 83.33 & 100 & 46 & 100 & 100 & 34 & 33.33 & 35 & 40 & 100 & 85 & 44 & 40 & 55 \\
\hline 50 & 100 & 100 & 48 & 100 & 90 & 47 & 100 & 100 & 43 & 16.67 & 55 & 49 & 83.33 & 90 \\
\hline Total & 90.33 & 95 & Total & 90.33 & 91.42 & Total & 81.67 & 87 & Total & 88.33 & 90.97 & Total & 88.66 & 83.97 \\
\hline
\end{tabular}

Destaca-se que o item 43 ('Eu gosto de brincar com ideias e de tentar pensar em como colocá-las em prática') foi o que mais apresentou discordâncias; apenas um juiz o classificou no padrão Intelectual, o restante o enquadrou no padrão Imaginativo. 0 item 2 ('Eu sou uma pessoa competitiva') foi outro que obteve concordância abaixo do esperado, somente três participantes o classificaram no padrão Intelectual. Quanto ao item 34 ('As palavras e os sons criam imagens inusitadas em minha mente'), apenas dois doutores o enquadraram no padrão esperado, os demais optaram pela SE Sensorial.

Os itens redigidos de forma negativa também apresentaram discordâncias entre os julgadores. No caso do item 38 ('Eu não sou sensível à cor, à forma e à textura das coisas como algumas pessoas são'), somente dois juízes o classificaram no padrão esperado, três participantes optaram pelo padrão Intelectual e um pelo Emocional. Já o item 44 ('Eu não sou uma pessoa emotiva'), foi classificado inadequadamente por três juízes.

\section{Aplicação piloto}

A aplicação do questionário, após as alterações resultantes das análises dos juízes doutores, revelou que estudantes do terceiro ano do Ensino Médio possuíam poucas dúvidas quanto aos termos usados. Mencionam-se, como exemplos de dúvidas, as palavras 'autônomo' do item 5 ['Eu sou um(a) pensador(a) autônomo'] e 'absorvente' do item 8 ('Observar a arte é uma experiência totalmente absorvente para mim'). No entanto, optou-se por não retirar ou substituir essas e outras palavras, uma vez que elas não prejudicaram a compreensão das frases para a grande maioria dos estudantes.

Optou-se somente por fazer alterações nos itens 38 e 44, que, anteriormente, eram: 'Eu não sou sensível (...)' e 'Eu não sou uma pessoa emotiva', passando a ser, respectivamente, 'Eu sou insensível (...)' e 'Eu sou uma pessoa não emotiva'. A opção por retirar a palavra 'não' ou por mudá-la de ordem foi decorrente do fato de alguns alunos apresentarem dúvidas para trabalhar com negativas ao responderem ao instrumento.

Optou-se, também, por modificar o item 2 ('Eu sou uma pessoa competitiva'), que passou a ser 'Eu sou uma pessoa competitiva em atividades físicas e/ou esportivas'. Essa alteração se deve tanto ao fato de muitos juízes o terem classificado em outros padrões que não o de origem quanto a uma percepção subjetiva de que os estudantes também tiveram essa compreensão.

\section{Análise por juízes mestrandos}

A análise do questionário feita por mestrandos permitiu, também, observar elevados índices de concordância, classificados de substanciais a quase perfeitos, uma vez que os valores obtidos por todos os juízes variaram entre .74 e .97 (com valores de $p<.001$ em todos os casos). 0 Quadro 2 apresenta a percentagem de concordância entre os juízes, obtida para cada item. Observou-se que, dos 50 itens, apenas seis apresentaram concordância abaixo da recomendada, ou seja, foram inferiores a $80 \%$. No total, os índices de concordância para os fatores ficaram acima desse valor. 
Quanto aos itens que apresentaram percentagens de concordância abaixo do esperado, convém destacar que: o item 9 ('Eu me preocupo muito'), originalmente vinculado ao padrão Emocional, foi classificado como Intelectual por 45\% $(n=9)$ dos juízes; o item 34 (As palavras e os sons criam imagens inusitadas em minha mente), pertencente ao padrão Imaginativo, foi elencado por $65 \%(n=13)$ dos sujeitos no padrão Sensorial; o item 38 ('Eu sou insensível à cor, à forma e à textura das coisas como algumas pessoas são'), do padrão Sensorial, foi classificado erroneamente por 30\% $(n=6)$ dos respondentes, sendo que cinco participantes o enquadraram no padrão Intelectual e um juiz o classificou no padrão Emocional; o item 41 ('Eu posso sentir uma mistura de emoções diferentes de uma só vez'), originalmente do padrão Emocional, foi classificado como Sensorial por 30\% $(n=6)$ dos participantes; o item 43 ('Eu gosto de brincar com ideias e tento pensar sobre como colocá-las em prática'), representante do padrão Intelectual, foi elencado erroneamente por $45 \%$ dos juízes $(n=9)$, sendo que oito mestrandos classificaram-no padrão Imaginativo e um juiz optou pelo padrão Sensorial; e o item 44 ('Eu sou uma pessoa não emotiva'), do padrão Emocional, também demostrou discordância entre $45 \%(n=9)$ dos juízes, sendo que oito optaram pela SE Intelectual e apenas um escolheu o padrão Sensorial.

\section{Retrotradução}

Após as análises e modificações descritas anteriormente, a retrotradução do instrumento foi enviada a um dos autores do OEQ-II - F. Falk (comunicação pessoal, oito de abril de 2011). Ele avaliou que a retrotradução dos itens apresentou significados compatíveis com a versão original. Chamou atenção apenas para o item 13, querendo se certificar de que a versão em português (13. Eu sinto muito prazer com o trabalho artístico dos outros) também faz referência à forte afinidade para a arte (13. I get great joy from the artwork of others). Como havia convergência de significado das duas versões e como a maioria dos juízes doutores não optou pela modificação da tradução, decidiu-se manter o item como originalmente sugerido.

\section{DISCUSSÃo}

Deve-se destacar que os procedimentos adotados nesta investigação se coadunam com os empregados em estudos que já efetuaram um processo de tradução do OEQ-II para outros idiomas. As traduções realizadas na Espanha, México e Jordânia, por exemplo, também contaram com uma aplicação piloto para a reformulação de itens (Al-Onizat, 2013; Falk et al., 2008; Sanz, 2006). Estudos de retrotraduções para verificar a convergência semântica também foram efetuadas na Espanha, Coréia do Sul e Jordânia (AlOnizat, 2013; Piirto et al., 2008; Sanz, 2006). No entanto, poucas pesquisas contaram com a participação de juízes especialistas no processo de obtenção de evidências de validade de conteúdo do OEQ-II; a versão para a Jordânia (Al-Onizat, 2013) é uma exceção.

Destaca-se que não há consenso na literatura quanto aos critérios para determinar o número ideal e a qualificação dos juízes na verificação da validade de conteúdo de um instrumento. Porém, é sensato afirmar que a seleção dos participantes deve levar em consideração a experiência e a qualificação dos membros (Grant \& Davis, 1997). A respeito da quantidade, há recomendações que variam entre cinco a 20 sujeitos (Alexandre \& Coluci, 2009). No caso do presente estudo, tanto a quantidade quanto a qualificação dos juízes doutores se enquadram no recomendado para tradução/adaptação de um instrumento, de modo que os seis participantes foram selecionados com base em suas publicações, formação e conhecimento da língua inglesa. Em geral, as análises de adequação dos itens realizadas pelos doutores demonstraram que o conteúdo da maioria dos itens é adequado para representar o padrão de SE a ser mensurado.

Apesar de, para muitos autores, a validade de conteúdo compreender somente a avaliação de um comitê de especialistas (Fitzner, 2007) ou de incluir, além dessa, o desenvolvimento ou tradução/adaptação do instrumento (Polit \& Beck, 2006), optou-se por apresentar, ademais, os resultados da aplicação preliminar da versão brasileira do OEQ-II. Tal opção se deve ao fato de que a compreensão dos itens por parte de amostras equivalentes à população-alvo também gera indicadores da qualidade semântica dos itens (Pasquali, 2010). No caso dessa medida, esse procedimento permitiu observar que parece adequado o autopreenchimento do instrumento por pessoas que tenham completado, pelo menos, o segundo ano do Ensino Médio.

Ressalta-se, também, que alguns autores recomendam a inclusão de pessoas leigas na avaliação (Alexandre \& Coluci, 2009), o que foi adotado no presente estudo ao utilizar mestrandos para classificar os itens em seus respetivos padrões. Apesar de estarem cursando uma pós graduação em Psicologia, pode se considerar que se trata de um grupo cego quanto à temática de SE. Esse tipo de colaborador permite, por exemplo, observar o quão clara é a relação do item com um fator, padrão etc., auxiliando no processo de obtenção de evidências de validade de conteúdo. As análises de adequação dos itens realizadas pelos 
mestrandos também demonstraram que o conteúdo de grande parte dos itens representa adequadamente o padrão de SE que pretende mensurar.

Deve-se ressaltar que a revisão da redação de alguns itens, resultante da análise efetuada pelos juízes doutores ou da aplicação preliminar, pareceu não acarretar mudanças significativas nos seus significados. 0 aprimoramento dos itens, principalmente os de número 2, 38 e 44, pode ser observado nos resultados obtidos na avaliação dos juízes mestrandos. Destaca-se que o fato de os itens 38 e 44 serem negativos pode ter contribuído para maior discordância entre os juízes, tanto doutores quanto mestrandos, já que necessitam de maior atenção no preenchimento.

Ressaltam-se ademais as divergências nas análises dos juízes no caso dos itens 34 e 43 . Em ambos os grupos - mestrandos e doutores -, houve uma tendência de os participantes os classificarem em dois padrões. Sendo assim, necessita-se de maior atenção a estes itens em análises posteriores, para verificar se, de fato, são representantes de dois ou mais padrões de SE.

Destaque deve ser dado também ao item 41, que, embora tenha sido classificado no padrão adequado - Emocional - por todas os juízes doutores, apresentou na análise por mestrandos algumas classificações no padrão Sensorial. Do mesmo modo, o item 9 também demonstrou baixos índices de concordância nas análises por juízes mestrandos, embora nas classificações observadas pelos juízes isso não tenha sido evidenciado. 0 fato de as classificações realizadas pelos doutores serem melhores para alguns itens do que as feitas pelos mestrandos, ainda que o percentual global de classificações corretas dos mestrandos seja, de modo geral, superior, pode ser decorrente do maior conhecimento que as primeiras possuem sobre sobredotação. Portanto, há que se considerar que, apesar de o emprego de juízes cegos ser recomendado pela literatura (Alexandre \& Coluci, 2009), esse procedimento pode representar um obstáculo na obtenção de evidências de validade de conteúdo quando se trata de um constructo complexo e pouco conhecido pelo senso comum.

\section{ESTUDO 2 - CONFIABILIDADE E EVIDÊNCIAS DE VALIDADE BASEADAS NA ESTRUTURA INTERNA DO OEQ-II}

\section{MÉTODO}

\section{Participantes}

Participaram deste estudo 281 estudantes universitários de uma instituição pública brasileira vinculados a seis cursos de graduação: Ciências da Computação ( $n=33$; 12.10\%); Enfermagem ( $n=21 ; 7.47 \%)$, Engenharia Civil $(n=59 ; 20.99 \%)$; Farmácia $(n=46 ; 16.37 \%)$, História $(n=45 ; 16.01 \%)$ e Psicologia $(n=$ 76; 27.05\%). A amostra foi composta por 152 estudantes do sexo feminino (54.09\%). A idade média em anos dos respondentes foi igual a $21.99(D P=4.88$; idade mínima = 17; idade máxima $=57)$.

\section{Instrumentos}

Foi utilizada a versão brasileira do OEQ-II alterada com base nos resultados do Estudo 1. Essa medida contém a mesma quantidade de itens que a versão original, pois as modificações foram realizadas apenas em seus enunciados, sendo mantida a forma de aplicação e correção.

\section{Procedimento}

Após o consentimento dos participantes, a aplicação do questionário foi efetuada em sala de aula. Ressalta-se que não foram estipulados critérios de exclusão para a amostra de estudantes universitários. Todos os presentes na sala de aula no momento da aplicação participaram da pesquisa, uma vez que não houve recusa quanto à participação. Não foi necessário excluir nenhum participante, por exemplo, por problemas de preenchimento (percentual elevado de respostas em branco) ou de outra natureza.

No que se refere aos procedimentos de análises dos dados, deve-se destacar que, para determinar a consistência interna dos padrões de SE, foi utilizado o coeficiente alfa de Cronbach. Neste caso, a literatura sugere como adequados índices superiores a .70 (Pasquali, 2003). As evidências de validade baseadas na estrutura interna foram obtidas por meio de Análise Fatorial Exploratória (AFE), adotando como método de extração a Fatoração de Eixos Principais com rotação ortogonal Varimax. A eleição por tais métodos deve-se às características de distribuição da amostra e às evidências teóricas de independência dos padrões (Ackerman, 2009). Destaca-se a opção por preestabelecer a fixação de cinco fatores a serem extraídos, convergindo com o modelo teórico de SE e considerando os resultados do Teste de Esfericidade de Bartlett e o Índice de Adequação da Amostra de Kaiser Meyer-Olkin (KMO) para a adequação da matriz de correlações. 


\section{Resultados}

Observou-se que todos os padrões medidos pela versão brasileira do OEQ-II obtiveram altos índices de confiabilidade: .80 (Psicomotor); .87 (Sensorial); .84 (Imaginativo); .86 (Intelectual); e .84 (Emocional). Verificou-se, também, que a retirada de itens não aumentaria expressivamente o alfa nos padrões de SE.

Com a AFE, confirmou-se a fatorabilidade da matriz de correlação dos itens da versão brasileira do OEQ-II por meio do KMO $=0.85$ e Teste de Esfericidade de Bartlett $\left(\chi^{2}(1225,281)=6460.45 ; p<.001\right)$. Extraíram-se, como previsto e solicitado, cinco fatores. Todos possuem valores próprios superiores a um e explicam conjuntamente $46.23 \%$ da variância total.

O Quadro 3 apresenta as cargas fatoriais obtidas pelos itens. Nela é possível perceber que o primeiro fator contém 12 itens, sendo que 10 correspondem ao padrão Sensorial, apresentando, em sua maioria, saturações mais altas $(.31<\lambda<.81)$. 0 segundo fator exibe 10 itens $(.47<\lambda<.71)-$ todos pertencentes ao padrão Intelectual. 0 terceiro contém nove itens - todos correspondentes ao padrão Psicomotor $(.40<\lambda<.79)$ - com exceção do item 21 , cuja carga fatorial $(\lambda=.31)$ foi exibida no Padrão Imaginativo. 0 quarto fator apresenta 11 itens, sendo que 10 deles representam o padrão Imaginativo $(.30<\lambda<.73)$. Por fim, o quinto fator exibe 11 itens, sendo 10 pertencentes ao padrão Emocional $(.43<\lambda$ $<.64)$.

Quadro 3. Resultado da análise factorial exploratório com o método de factoração do eixo principal (Rotação Varimax).

\begin{tabular}{|c|c|c|c|c|c|c|c|c|c|}
\hline \multicolumn{2}{|c|}{$\begin{array}{c}\text { Fator } 1^{a} \\
\text { (Sensorial) }\end{array}$} & \multicolumn{2}{|c|}{$\begin{array}{c}\text { Fator } 2^{b} \\
\text { (Intelectual) }\end{array}$} & \multicolumn{2}{|c|}{$\begin{array}{c}\text { Fator } 3^{c} \\
\text { (Psicomotor) }\end{array}$} & \multicolumn{2}{|c|}{$\begin{array}{c}\text { Fator } 4^{\mathrm{d}} \\
\text { (Imaginativo) }\end{array}$} & \multicolumn{2}{|c|}{$\begin{array}{c}\text { Fator 5e } \\
\text { (Emocional) }\end{array}$} \\
\hline Itens & $\lambda$ & Itens & $\lambda$ & Itens & $\lambda$ & Itens & $\lambda$ & Itens & $\lambda$ \\
\hline 45 & .81 & 36 & .71 & 50 & .79 & 24 & .73 & 49 & .64 \\
\hline 3 & .69 & 30 & .67 & 15 & .74 & 22 & .68 & 35 & .63 \\
\hline 13 & .67 & 43 & .63 & 39 & .69 & 14 & .64 & 44 & .62 \\
\hline 32 & .66 & 16 & .60 & 2 & .65 & 47 & .62 & 6 & .59 \\
\hline 8 & .63 & 5 & .59 & 18 & .64 & 1 & .58 & 31 & .58 \\
\hline 48 & .57 & 19 & .59 & 10 & .63 & 20 & .57 & 17 & .51 \\
\hline 37 & .57 & 40 & .53 & 29 & .57 & 4 & .54 & 11 & .50 \\
\hline 46 & .55 & 25 & .52 & 7 & .42 & 34 & .41 & 41 & .47 \\
\hline 27 & .47 & 12 & .50 & 42 & .40 & 21 & .31 & 26 & .44 \\
\hline 34 & .45 & 23 & .47 & & & 33 & .30 & 9 & .43 \\
\hline 49 & .34 & & & & & 28 & .30 & 37 & .33 \\
\hline 38 & .31 & & & & & & & & \\
\hline
\end{tabular}

Nota: Variância Explicada, Valores próprios: a9.33\%, 9.95; b8.39\%, 4.50; ‘6.04\%, 3.59; d7.71\%, 2.89; e7.49\%, 2,17.

Três itens (34, 37 e 49), além de se mostrarem associados aos padrões originalmente propostos pelos autores do OEQ-II, também se associaram a outros padrões. Porém, as cargas fatoriais nestes foram inferiores àqueles, exceto no caso do item 34 , que obteve carga maior no padrão Sensorial $(\lambda=.45)$ e não em seu padrão de origem, ou seja, Imaginativo $(\lambda=.41)$.

\section{DISCUSSÃO}

No que diz respeito às análises da consistência interna da versão em português do OEQ-II, observaram-se altos índices de confiabilidade para os padrões de SE medidos pelo instrumento. Os valores dos alfas de Cronbach são próximos ou, em alguns casos, superiores aos encontrados em pesquisas que utilizaram o OEQ-II em outros países e idiomas (Al-Onizat, 2013; Falk et al., 2008; Piirto et al., 2008; Sanz, 2006; Siu, 2010; Tieso, 2007a; Warne, 2011b).

Os resultados obtidos com a AFE revelam que a maioria dos itens tende a se agrupar no fator esperado, isto é, junto com os outros itens do seu padrão. Assim, observa-se que a versão em português do OEQ-II também parece ter uma estrutura multidimensional com cinco fatores. Essa constatação corrobora os resultados de outras pesquisas (Falk et al, 1999; Sanz, 2006; Warne, 2011a) e a teoria que fundamenta o questionário (Dabrowski, 1972).

A fim de estabelecer uma associação com o estudo anterior, é preciso destacar que as alterações efetuadas no Estudo 1 serviram para melhorar a compreensão dos itens ou para aproximá-los ainda mais de seu sentido original. 0 aprimoramento dos itens 2, 38 e 44, também puderam ser observados na AFE, uma vez que, apesar de se mostrarem problemáticos nas análises por juízes, apresentaram saturações somente nos fatores em que eram esperados.

Ao considerar outros itens que apresentaram divergências nas análises dos juízes, a AFE revelou que somente o 34 satura em dois padrões de SE: Imaginativo e Sensorial. Assim, parece que, de fato, esse 
item pode ser representante dos dois fatores. A afirmativa 'As palavras e os sons criam imagens inusitadas em minha mente' denota tanto uma capacidade imaginativa quanto o uso dos sentidos.

\section{ESTUDO 3 - EVIDÊNCIAS DE VALIDADE BASEADAS NAS RELAÇÕES COM INSTRUMENTOS QUE INVESTIGAM CONSTRUCTOS ANÁLOGOS AO OEQ-II}

\section{MÉTODO}

\section{Participantes}

A amostra deste estudo foi composta por 368 universitários provenientes da mesma instituição do Estudo 2, sendo que 242 participantes são do sexo feminino (65.76\%). Os estudantes apresentaram média de idade em anos de $22.80(D P=7.01$; idade mínima $=17$; idade máxima $=69)$ e pertenciam às seguintes graduações: Física $(n=21 ; 5.71 \%)$, Fisioterapia $(n=29 ; 7.88 \%)$, Serviço Social $(n=43$; $11.68 \%)$, Filosofia $(n=44 ; 11.98 \%)$, Artes e Design $(n=44 ; 11.98 \%)$, Arquitetura e Urbanismo $(n=49$; $13,32 \%)$, Educação Física $(n=60 ; 16.30 \%)$ e Psicologia $(n=78 ; 21.20 \%)$.

\section{Instrumentos}

Além da versão em português do OEQ-II, tal como utilizada no Estudo 2, os estudantes de graduação responderam à Avaliação dos Tipos Profissionais de Holland (ATPH) (Primi, Muniz, Nunes, \& Murgo, 2008) e aos 10 itens do fator Atividade/Passividade das Escalas de Personalidade de Comrey (CPS) (Costa, 2009). O ATPH, composto por 154 itens em uma escala tipo Likert de quatro pontos, tem como objetivo avaliar os seis tipos de personalidades vocacionais: realista, investigador, artístico, social, empreendedor e convencional. Estudos têm demonstrado boas evidências de validade e fidedignidade do instrumento (Mansão \& Noronha, 2011; Mansão, Noronha, \& Ottati, 2011; Noronha, Mansão, Silva, Freitas, \& Pereira, 2013). O fator Atividade/Passividade das CPS investiga aspectos relacionados à disposição para atividades físicas, gasto de energia, resistência etc. Este instrumento é, atualmente, recomendado para uso pelo SATEPSI (CFP, 2013). A autorização para a retirada e utilização dos itens foi concedida tanto pelo autor (F. R. Costa, comunicação pessoal, 19 de abril de 2011) da escala, quanto pela VETOR Editora (comunicação pessoal, 17 de março de 2011), que é a detentora oficial dos direitos autorais do instrumento.

É preciso destacar que, devido ao fato de no Brasil não haver instrumentos que meçam as formas de SEs, a validade convergente-discriminante fica limitada. Sendo assim, optou-se por utilizar instrumentos que medem constructos análogos às características de personalidade daqueles que possuem altos níveis de SE, como é o caso do ATPH (Primi et al., 2008), e da CPS (Costa, 2009), descritos anteriormente.

\section{Procedimento}

A aplicação do OEQ-II seguiu os mesmos procedimentos do Estudo 1. Ao final, os estudantes também responderam ao ATPH e aos 10 itens do fator Atividade/Passividade da CPS. Para a análise dos dados, utilizou-se o coeficiente de correlação Pearson.

\section{Resultados}

O Quadro 4 apresenta as correlações entre os padrões de SE e as tipologias da ATPH. O padrão Sensorial apresentou correlações: positiva, significativas e moderadas com o Tipo Artístico e positivas e significativas, porém fraca, com o Tipo Realista. A medida de SE Imaginativa correlacionou-se positiva e significativamente de forma fraca com as tipologias Artística e Realista. 0 padrão Intelectual obteve correlação positiva, significativa e fraca com os Tipos Investigativo, Realista, Artístico, Convencional e Empreendedor. 0 padrão de SE Emocional correlacionou-se positiva e significativamente de forma fraca com a tipologia Social, Artística e Empreendedora.

Quadro 4. Correlações entre os escores da versão em português do OEQ-II com os factores da ATPH e da CPS.

\begin{tabular}{llccccc}
\hline & Instrumentos & Psicomotor & Sensorial & $\begin{array}{c}\text { Padrões de SE } \\
\text { Imaginativo }\end{array}$ & Intelectual & Emocional \\
\hline ATPH & Realista & $.17^{* *}$ & $.17^{* *}$ & $.11^{*}$ & $.27^{* *}$ & .01 \\
& Investigativo & $.20^{* *}$ & .04 & -.02 & $.38^{* *}$ & .01 \\
& Artístico & .02 & $.63^{* *}$ & $.38^{* *}$ & $.36^{* *}$ & $.25^{* *}$ \\
& Social & $.11^{*}$ & .06 & .06 & .08 & $.35^{* *}$ \\
& Empreendedor & $.25^{* *}$ & .01 & .02 & $.12^{*}$ & $.13^{*}$ \\
& Convencional & $.14^{* *}$ & -.08 & -.05 & $.16^{* *}$ & -.04 \\
CPS & Atividade/Passividade & $.60^{* *}$ & -.07 & $-.20^{* *}$ & $.11^{*}$ & .02 \\
\hline${ }^{*} p<.05 ;{ }^{* *} p<.01$ & & & & &
\end{tabular}


Os escores da subescala Passividade/Atividade da CPS (Quadro 4) demonstraram correlação positiva significativa moderada com o padrão Psicomotor e fraca com o padrão Intelectual. Já o padrão Imaginativo apresentou correlação negativa e significativamente fraca com essa escala.

\section{DISCUSSÃO}

As evidências de validade convergente-discriminante do OEQ-II são uma das principais limitações psicométricas do instrumento (Carman, 2011; Oliveira \& Barbosa, 2014). Reitera-se que poucos estudos procuraram associar essa medida com instrumentos análogos. Embora muitas investigações (p. ex. Broeck, Hofmans, Cooremans, \& Staels; Siu, 2010) utilizem testes de inteligência para a identificação ou categorização de amostras de sobredotados, são poucas as que tiveram como objetivo correlacionar SE Intelectual com tais medidas (Carman, 2011). Entretanto, alguns estudos relatam correlações positivas entre o OEQ-II e outros instrumentos. Reffel (2012), por exemplo, encontrou correlações positivas e significativas entre uma medida de criatividade e os padrões de SE, inclusive a SE Imaginativa. Também foram obtidas correlações positivas e significativas entre os escores do Sensory Profile e as SEs Sensorial e Psicomotora (Carman, 2011).

Observou-se, no presente estudo, que as médias apresentadas nos padrões de SE associaram-se com as pontuações obtidas pelas tipologias de personalidades semelhantes. Destacam-se as correlações entre os padrões Sensorial e Imaginativo com o Tipo Artístico, o padrão Intelectual com a Tipologia Investigativa e o padrão Emocional com o Tipo Social da ATPH. A correlação entre o padrão Psicomotor e a subescala Atividade/Passividade da CPS, também merece destaque.

As associações relatadas anteriormente, mesmo com magnitudes fracas ou moderadas, eram esperadas, tendo em vista que os constructos investigados não são os mesmos, mas há semelhanças entre os padrões de SE medidos pelo OEQ-II e as descrições de algumas tipologias do ATPH (Primi et al., 2008) e da subescala da CPS (Costa, 2009). No último caso, por exemplo, há uma evidente aproximação teórica entre o que se denomina SE Psicomotora no OEQ-II e o que a escala CPS conceitua Atividade/Passividade. Há itens para a Atividade que analisam características como agitação, energia física e gosto por estar em movimento (Costa, 2009). Estes aspectos podem ser considerados indicadores de SE Psicomotora (Dabrowski, 1996). Associações teóricas desse tipo também podem ser observadas nas definições das tipologias do ATPH com alguns padrões de SE. Cita-se, como exemplo, a Tipologia Investigativa que se destaca em pessoas analíticas, críticas e que possuem uma forte necessidade de compreender as coisas (Noronha et al., 2013). Estas, entre outras características, são semelhantes às encontradas em pessoas com SE Intelectual. Nesse sentido, os resultados apresentados no presente estudo asseveram mais evidências de validade do OEQ-II.

\section{DISCUSSÃO GERAL}

Este texto apresenta as primeiras evidências de validade da versão brasileira de uma medida de SE. Assevera-se que as estratégias utilizadas para a tradução e obtenção de evidências de validade baseadas no conteúdo, na estrutura interna e na relação com instrumentos que investigam constructos análogos foram equivalentes às sugeridas pela literatura (Alexandre \& Coluci, 2009; American Educational Research Association, American Psychological Association, \& National Council on Measurement in Education, 1999) e às recomendadas pelo SATEPSI (CFP, 2003, 2010).

Em resumo, os três estudos apresentados no texto denotam que a versão brasileira do OEQ-II possui propriedades psicométricas adequadas. No Estudo 1, apesar de poucos itens se mostrarem mais problemáticos, de modo geral, tanto o grau de concordância e a precisão entre juízes - doutores e mestres - quanto a aplicação piloto mostraram-se bastante satisfatórias. A equivalência entre o original em inglês e a versão retrotraduzida denota semelhança conceitual e semântica entre os itens das duas versões. Esses resultados geraram boas evidências de validade de conteúdo da medida. 0 Estudo 2 corroborou a estrutra multidimensional do OEQ-II, composta por cinco fatores, com grande parte dos itens saturando nos padrões esperados. Além disso, bons índices de confiabilidade puderam ser observados. 0 Estudo 3, por fim, identificou correlações positivas significativas com instrumentos que medem constructos análogos, evidenciando boas evidências de validade baseadas nas relações com variáveis externas. Assim, os resultados do processo inicial de obtenção de evidências de validade e confiabilidade da versão brasileira do OEQ-II podem ser considerados satisfatórios.

Não obstante o processo rigoroso de adaptação para a língua portuguesa do Brasil, algo que tem sido enfatizado por diversos pesquisadores (Borsa, Damásio, \& Bandeira, 2012; Cardoso, 2006), e as evidências de validade e confiabilidade terem sido satisfatórias, são necessários estudos adicionais sobre as propriedades psicométricas da versão brasileira do OEQ-II, especialmente para os itens que se mostraram mais problemáticos. Dentre outras fontes de evidência de validade que lançam luz sobre a organização dos itens em fatores, sugere-se a realização de Análise Fatorial Confirmatória. Recomendam- 
se, também, estudos que verifiquem outros tipos de evidências de validade (por exemplo, validade de critério) e que contem com amostras mais amplas e mais representativas da população brasileira. Do mesmo modo, também se torna necessário que o questionário seja aplicado e analisado na população alvo à qual se destina, ou seja, indivíduos com características de sobredotação, e correlacionado com medidas que sejam utilizadas para essa finalidade.

Recomenda-se, ademais, que, para estudos futuros, sejam mantidos todos os itens do instrumento, até que se analisem mais profundamente outras propriedades psicométricas. Assim, deve ser empregada a mesma versão utilizada nos estudos 2 e 3 com 50 itens subdivididos em cinco padrões de SE.

Salienta-se, mais uma vez, que a versão brasileira do OEQ-II pode auxiliar o aprimoramento da identificação de indivíduos com sobredotação, uma vez que a escassez de medidas válidas e confiáveis em língua portuguesa e para esse objetivo é notória (Barbosa et al., 2012). Ressalta-se, novamente, que esse instrumento pode ajudar na compreensão de aspectos que atualmente estão sendo pouco investigados como, por exemplo, as características sensoriais, emocionais, imaginativas e psicomotoras. 0 estudo das forças e expressões das SE dessas pessoas, além de servir para obtenção de mais informações sobre as suas características, pode, consequentemente, servir como base para planejar e implantar programas de atendimento, auxiliando o processo que almeja transformar dotação em talento.

\section{Referências}

Ackerman, C. M. (1997). Identifying gifted adolescents using personality characteristics: Dabrowski's overexcitabilities. Roeper Review, 19, 229-236.

Ackerman, C. M. (2009). The essential elements of Dabrowski's theory of positive disintegration and how they are connected. Roeper Review, 31(2), 81-95.

Alexandre, N. M. C., \& Coluci, M. Z. O. (2009). Validade de conteúdo nos processos de construção e adaptação de instrumentos de medidas. Ciência e saúde coletiva, 16(7), 3061-3068.

Al-Onizat, S. H. (2013). The psychometric properties of a Jordanian version of Overexcitability Questionnaire-Two, OEQII. Criative Education, 4(1), 49-61.

American Educational Research Association, American Psychological Association \& National Council on Measurement in Education. (1999). Standards for educational and psychological testing. Washington, DC: AERA.

Barbosa, A. J. G., Schelini, P. W., \& Almeida, L. C. (2012). Medidas de dotação e talento: produção científica em Psicologia (2006 - 2011). In E. Boruchovitch, A. A. A. Santos, \& E. Nascimento (Orgs.), Avaliação psicológica nos contextos educativo e psicossocial (pp. 33-52). São Paulo: Casa do Psicólogo.

Borsa, J. C., Damásio, B. F., \& Bandeira, D. R. (2012). Adaptação e validação de instrumentos psicológicos entre culturas: Algumas considerações. Paidéia, 22(53), 423-432.

Bouchar, L. L. (2004). An instrument for the measure of dabrowskian overexcitabilities to identify gifted elementary students. Gifted Child Quarterly, 48(4), 339-350.

Bouchet, N., \& Falk, R. F. (2001). The relationship among giftedness, gender, and overexcitability. Gifted Child Quarterly, 45(4), 260-267.

Broeck, W. V., Hofmans, J., Cooremans, S., \& Staels, E. (2013). Factorial validity and measurement invariance across intelligence levels and gender of the Overexcitabilities Questionnaire-II (OEQ-II). Psychological Assessment, 30, 1-14.

Cardoso, I. (2006). Aspectos transculturais na adaptação de instrumentos de avaliação psicológica. Interacções, 10, 98-112.

Carman, C. A. (2011). Adding personality to gifted identification: Relationships among traditional and personality-based constructs. Journal of Advanced Academics, 22, 412-446.

Carroll, J. B. (1993). Human cognitive abilities: A survey of factor-analytic studies. Cambridge, UK: Cambridge University Press.

Chang, H. J., \& Kuo, C. C. (2009). Overexcitabilities of gifted and talented students and its related researches in Taiwan. Asia-Pacific Journal of Gifted and Talented Education, 1(1), 41-74.

Conselho Federal de Psicologia (2003). Resolução CFP $n^{\underline{o}}$ 002/2003. Disponível em http://www.crp07.org.br/upload/legislacao/legislacao47.pdf. Acesso em 02 de outubro de 2013.

Conselho Federal de Psicologia. (2010). Avaliação psicológica: Diretrizes na regulamentação da profissão. Brasília: Conselho Federal de Psicologia.

Conselho Federal de Psicologia (2013). Sistema de avaliação de testes psicológicos: Testes psicológicos aprovados para uso. Disponível em http://www.pol.org.br/satepsi/sistema/admin.cfm?lista1=sim. Acesso em 02 de outubro de 2013.

Costa, F. R. (2009). CPS: Escalas de personalidade de Comrey (3a ed.). São Paulo: Vetor.

Dabrowski, K. (1972). Psychoneurosis is not an illness: Neuroses and psychoneuroses from the perspective of positive disintegration. London: Gryf. 
Dabrowski, K. (1996). Multilevelness of emotional and instinctive functions. Lublin, Poland: Towarzystwo Naukowe Katolickiego Uniwersytetu Lubelskiego.

Dabrowski, K., Kawczak, A., \& Piechowski, M. (1970). Mental growth through positive disintegration. London: Gryf.

Diniz, A. M., Almeida, L. S., \& Pais, L. G. (2007). Contextos profissionais e práticas da avaliação psicológica: Inquérito aos psicólogos portugueses. Psico-USF, 12(1), 1-12.

Dunn, W. (1997). The impact of sensory processing abilities on the daily lives of young children and their families: A conceptual model. Infants and Young Children, 9, 23-35.

Falk, R. F., Lind, S., Miller, N. B., Piechowski, M. M., \& Silverman, L. K. (1999). The Overexcitability Questionnaire Two (OEQ II). Manual, scoring system, and questionnaire. Denver: Institute for the Study of Advanced Development.

Falk, R. F., Yakmaci-Guzel, B., Chang, A. H., Sanz, R. P. S., \& Chavez-Eakle, R.A. (2008). Measuring overexcitabily: Replication across five countries. In S. Mendaglio (Org.), Dabrowski's theory of positive disintegragion (pp. 183-199). Scottsdale AZ: Great Potential Press, Inc.

Feldhusen, J. F. (1998). Talent development, expertise, and creative achievement. Paper presented at the American Psychological Association Annual Convention, San Francisco, CA.

Fitzner, K. (2007). Reliability and validity. The Diabetes Educator, 33(5), 775-780.

Gagné, F. (1985). Giftedness and talent: Reexamining a reexamination of the definitions. Gifted Child Quarterly, 29(3), 103-112.

Gagné, F. (2004). Transforming gifts into talents: The DMGT as a developmental theory. High ability studies, 15(2), 119-147.

Grant, J. S., \& Davis L. L. (1997). Selection and use of content experts for instrument development. Research in Nursing \& Health, 20(3), 269-274.

Gross, C. M., Rinn, A. N., \& Jamieson, K. M. (2007). Gifted adolescents' overexcitabilities and self-concepts: An analysis of gender and grade level. Roeper Review, 29(4), 240-248.

Guenther, Z. C. (2006a). Dotação e talento: Reconhecimento e identificação. Revista Educação Especial, 28, 195-208.

Guenther, Z. C. (2006b). Desenvolver capacidades e talentos: Um conceito de inclusão. Petrópolis, RJ: Vozes.

Heller, K. A., Perleth, C., \& Lim, T. K. O. The Munich model of giftedness designed to identigy and promote gifted students. In R. J. Sternberg \& J. Davidson (Eds.), Conceptions of giftedness (pp. 174-188). Cambridge: Cambridge University Press.

Kaufman, S. B., \& Sternberg, R. J. (2008). Conceptions of giftedness. In S. I. Pfeiffer (Ed.), Handbook of giftedness in children (pp. 71-91). Tallahassee, FL: Springer.

Landis, J. R., \& Koch, G. G. (1977). The measurement of observer agreement for categorical data. Biometrics, 33, 159-74.

Lysy, K. Z., \& Piechowski, M. M. (1983). Personal growth: An empirical study using Jungian and Dabrowskian measures. Genetic Psychology Monographs, 108, 267-320.

Mansão, C. S. M., \& Noronha, A. P. P. (2011). Avaliação dos tipos profissionais de Holland: Verificação da estrutura interna. Revista de Psicología, 13(1), 46-58.

Mansão, C. S. M., Noronha, A. P. P., \& e Otatti, F. (2011). Interesses profissionais: Análise correlacional entre dois instrumentos de avaliação. Revista Brasileira de Orientação Profissional, 12(2), 175-183.

Noronha, A. P. P., Mansão, C. S. M., Silva, M. A., Freitas, P. C. S., \& Pereira, G. O. A. (2013). Evidências de validade convergente-discriminante para a avaliação dos tipos profissionais de Holland (ATPH). Psicologia: Ciência e Profissão, 33(1), 04-15.

Noronha, A. P. P., \& Reppold, C. T. (2010). Considerações sobre a avaliação Psicológica no Brasil. Psicologia: Ciência e Profissão, 30, 192-201.

Noronha, A. P. P., Primi, R., \& Alchieri, J. C. (2005). Instrumentos de avaliação mais conhecidos/utilizados por psicólogos e estudantes de psicologia. Psicologia: Reflexão e Crítica, 18(3), 390-401.

Oliveira, E. P. L. (2007). Alunos sobredotados: A aceleração escolar como resposta educativa. Tese de doutorado. Braga, Portugal: Universidade do Minho - Pós-graduação em Psicologia da Educação.

Oliveira, J. C., \& Barbosa, A. J. G. (2014). Instrumentos de sobre-excitabilidade: Uma revisão sistemática. Arquivos Brasileiros de Psicologia, 66(1), 117-134.

Pasquali, L. (2001). Técnicas de exame psicológico - TEP: manual. São Paulo: Casa do Psicólogo/Conselho Federal de Psicologia.

Pasquali, L. (2003). Psicometria: Teoria dos testes na psicologia e educação. Petrópolis: Vozes.

Pasquali, L. (2010). Instrumentação psicológica. Porto Alegre: Artes Médicas.

Piechowski, M. M. (1975). A theoretical and empirical approach to the study of development. Genetic Psychology Monographs, 92, 231-297. 
Piechowski, M. M. (1979). Developmental potential. In N. Colangelo, \& R. Zaffrann (Orgs.), New voices in counseling the gifted (pp. 25-57). Dubuque, Iowa: Kendall Hunt.

Piechowski, M. M. (1996). Overexcitability Questionnaire Revised (OEQ-REV). In S. Mendaglio (Org.), Dabrowski's theory of positive disintegration (pp. 269-270). Scottsdale AZ: Great Potential Press, Inc.

Pierson, E. E., Kilmer, L. M. Rhlisberg, B. A., \& McIntosh, D. E. (2012). Use of brief intelligence tests in the identification of giftedness. Journal of Psychoeducational Assessment, 30(1), 10-24.

Piirto, J., Montgomery, D., \& May, J. (2008). A comparison of Dabrowski's overexcitabilities by gender for American and Korean high school gifted students. High Ability Studies, 19(2), 141-153.

Polit, D. F., \& Beck, C. T. (2006). The content validity index: are you sure you know what's being reported? Critique and recomendationas. Research in Nursing \& Health, 29, 489-497.

Primi, R., Muniz, M., Nunes, M. F. O., \& Murgo, C. M. (2008). Avaliação dos Tipos Profissionais de Holland. Itatiba, SP: Autor.

Reffel, J. A. (2012). Overexcitability and creative perception. Poster session presented at the annual meeting of the National Association for Gifted Children, Denver, CO.

Renzulli, J. S. (1978). What makes giftedness? Reexamining a definition. Phi Delta, 60, 180-184.

Renzulli, J. S. (1999). What is this thing called giftedness, and how do we develop it? A twenty five year perspective. Journal for the Education of the Gifted, 23(1), 3-54.

Renzulli, J. S. (2005). The three ring conception of giftedness: A developmental model for creative productivity. In R. J. Sternberg \& J. Davidson (Eds.), Conceptions of giftedness (pp. 246-279). Cambridge: Cambridge University Press.

Sanz, R. P. S. (2006). El alumno superdotado y sus problemas de aprendizaje: Validación del OEQ-II como prueba de diagnostic. Tese de doutorado. Madrid, Espanha: Universidad Complutense de Madrid Pós-graduação em Educação.

Simões, M. R. (2005). Potencialidades e limites do uso de instrumentos no processo de avaliação psicológica. Psicologia, Educação e Cultura, 9(2), 237-264.

Siu, A. F. Y. (2010). Comparing overexcitabilities of gifted and non-gifted school children in Hong Kong: Does culture make a difference? Asia Pacific Journal of Education, 30(1), 71-83.

Soares, A. M. I., Souza Arco-Verde Y. F., \& Baibich, T. M. (2004). Superdotação: Identificação e opções de atendimento. Educar em Revista, 23, 125-141.

Tieso, C. L. (2007a). Overexcitabilities: A New Way to Think About Talent? Roeper Review, 29(4), 232-239.

Tieso, C. L. (2007b). Patterns of overexcitabilities in identified gifted students and their parents: A hierarchical model. Gifted Child Quarterly, 51(1), 11-22.

Virgolim, A. M. R. (1997). O indivíduo superdotado: História, concepção e identificação. Psicologia: Teoria e Pesquisa, 13(1), 173-183.

Warne, R. T. (2011a). An investigation of measurement invariance across genders on the Overexcitability Questionnaire - Two. Journal of Advanced Academics, 22(4) 578-593.

Warne, R. T. (2011b). A reliability generalization of the Overexcitability Questionnaire -Two. Journal of Advanced Academics, 22(5), 671-692.

Wirthwein, L., \& Rost, D. H. (2011). Focussing on overexcitabilities: Studies with intellectually gifted and academically talented adults. Personality and Individual Differences, 51, 337-342.

Historial do artigo

Recebido $11 / 07 / 2014$

Aceite $\quad 30 / 10 / 2014$

Publicado 12/2014 
Tradução, adaptação e evidências de validade do OEQ-II 